\title{
DNA tracking within a nanochannel: device fabrication and experiments
}

\author{
V. R. S. S. Mokkapati, ${ }^{* a}$ V. Di Virgilio, ${ }^{b}$ C. Shen, ${ }^{a}$ J. Mollinger, ${ }^{a} \mathrm{~J}_{\text {. Bastemeijer }}{ }^{a}$ and A. Bossche $^{a}$ \\ Received 27th January 2011, Accepted 2nd June 2011 \\ DOI: 10.1039/c1lc20075e
}

\begin{abstract}
Fabrication of nanochannels is drawing considerable interest due to its broad applications in nanobiotechnology (e.g. biomolecular sensing and single DNA manipulation). Nanochannels offer distinct advantages in allowing a slower translocation and multiple sensing spots along the channel, both of which improve the read-out resolution. However, implementing electrodes inside the nanochannel has rarely been demonstrated to our knowledge. The device described in this work is a Si-Glass anodically bonded Lab-on-a-Chip (LOC) device of a few millimetres in size capable of performing DNA manipulation. The LOC device structure is based on two mainstream microchannels interconnected by nanochannels. DNA, once trapped within the nanochannel, has been tracked throughout the length of the channel and the data have been recorded and analysed.
\end{abstract}

\section{Introduction}

Nanofluidics is the study of behavior, manipulation and control of fluids that are confined to nanometre dimensions (typically 1$100 \mathrm{~nm}$ ). The behavior of fluids confined in these structures is different from those of the micrometre range because the characteristic physical scaling lengths of the fluid (e.g.: debye length, hydrodynamic radius) closely coincide with the dimensions of the nanostructure itself. ${ }^{1}$ Transport in the nanochannels is the first and foremost important phenomenon which includes flow detection, liquid transport, control of molecular transport and separation techniques. Insight into all these aspects is quite important in order to progress further successfully in nanofluidics. ${ }^{1}$

Fluid interactions with the walls of the nanochannels are most prominent because of the large surface to volume ratio. ${ }^{1,2}$ Effects posed by the channel walls will certainly influence the transport of the particles within a nanochannel and the best example to determine these effects is the occurrence of EDL (electrical double layer). Aside from the EDL, it was reported in the literature that the surface roughness will strongly affect the EOF (electro-osmotic flow) and reduce the velocity of the liquid to a great extent. ${ }^{3}$ Nanochannel walls are in general negatively charged and therefore the particles that are to be induced should also be negatively charged to prevent clogging within the channel. ${ }^{1}$ If positively charged particles are injected, their adsorption to the wall decreases the EOF by decreasing the wall potential.

\footnotetext{
${ }^{a}$ Department of Microelectronics, Bio-devices group, Delft University of Technology/DIMES, Mekelweg 4, 2628 CD, The Netherlands

${ }^{b}$ Department d'Ingenyeria Electronica, Universitat Politecnica de Catalunya Campus Nord, ClJordi Girona 1-3, 08034 Barcelona, Spain. E-mail:v.r.s.s.mokkapati@tudelft.nl
}

The length of the nanochannel and the material on which it is fabricated define the fabrication procedure of the nanochannel. Enormous research that has been carried out and the unique physical and chemical properties make silicon the most opted for material. Other than silicon, the next best option would be a polymer because of its low processing costs and feasibility to produce disposable nanofluidic devices.

Anodic bonding is a common technique in nanochannel fabrication because of the homogeneity required by long channels. ${ }^{2,45}$ Researchers have also reported nanochannels fabricated by sacrificial etching which avoids the bonding and planarization procedures. ${ }^{6,7}$

Nanofluidics has a significant impact on medicine and clinical diagnostics with the development of LOC devices for diagnostics and related techniques (separation and determination of biomolecules like DNA and proteins, selective separation and transfer of analytes depending on the size and mass, etc. $)^{2}$ Nanofluidics is also applicable in forensics, explosive detection, wastewater treatment, pollution control, food processing, etc. ${ }^{8}$

\section{Motivation}

Fabrication of nanochannels is drawing considerable interest due to its broad applications in nanobiotechnology (e.g. biomolecular sensing and single DNA manipulation) and in fundamental studies of nanofluidic physical phenomena. Although the fabrication of inorganic nanopores, mostly driven by the application of DNA sequencing and biosensing, has become relatively mature, nanochannels offer distinct advantages over nanopores in allowing a slower translocation and multiple sensing spots along the channel, both of which improve the read-out resolution. ${ }^{20}$

The main aim behind fabricating these nanofluidic devices with integrated electrodes and nearly flat nanochannels is to study the behavior of biomolecules like DNA within a localized 
electric field. The behavior of the particles in response to localized electrodes will be different from the response to the beginend electrode configuration. Implementing electrodes inside the nanochannels has rarely been demonstrated to our knowledge.

The fabricated nanochannels were tested by filling them initially with ethanol (ethanol reduces the surface tension) followed by rhodamine + ethanol + di-water. Further to study the working of the electrodes, fluorescent polystyrene beads of 200 $\mathrm{nm}$ were injected through the inlet of the microchannel. By switching on the electrodes, the beads were diverted into the nanochannels by negative di-electrophoresis. The same principle can be used to divert and uncoil the DNA within the nanochannel, as DNA carries a net negative charge. This device can also be used to study the separation of bio-molecules depending on their size and their behavior in a localized electric field within flat nanochannels.

The above research often makes use of nanofluidic devices to study particles in a nanochannel confinement. Most devices however have the electrical connections outside the nanochannels which largely limit the possibilities of particle manipulation. The devices introduced in this paper have electrodes embedded in the walls of the nanochannels and add more degrees of freedom for manipulating flow profiles and particle behavior in nanochannels.

\section{Design of the device}

The basic design of the nanofluidic device is presented here. Simulation results and practical considerations were taken into account while designing the geometry of the electrodes as well as the nanochannels.

The working device has two microchannels $300 \mu \mathrm{m}$ wide, interconnected with several nanochannels (Fig. 1). Nanochannels were designed with two different widths (50 and $15 \mu \mathrm{m}$ ) but of the same length ranging from $550-750 \mu \mathrm{m}$. The idea of having two different widths is to study the behavior of the particles in both the cases as the width of the channels also has an effect on the behavior of the particles. Each nanochannel has

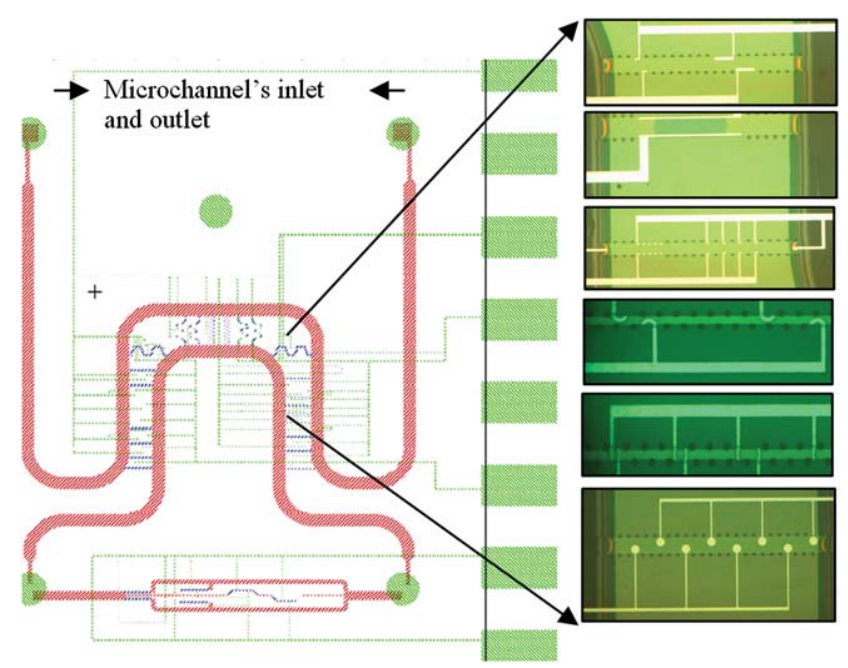

Fig. 1 Design of the working device, nanochannels bridge the two microchannels. Embedded electrode configurations within different nanochannels can also be observed. a different electrode configuration of different shapes and sizes. Photographs of some nanochannels with different configurations can be seen in Fig. 1. The embedded electrode standards are insulated from the liquid by an $100 \mathrm{~nm}$ thick silicon dioxide layer and can be used to generate local dielectrophoretic forces acting on the particles in the channel, as well as to induce local alterations to the electrical double layer.

The electrodes can also be exposed to the liquid by local removal of the insulation layer and can be used to change the potential and electric fields in the channel directly.

\section{Simulations}

Even though some simple simulation results were carried out, the design of this device, electrode configuration, lengths and widths of channels and the prospective depths are mainly based on practical considerations.

The transition from the micro- to nanorange produces some interesting effects on the fluids and on the biological samples behavior. Decreasing the cross-section of the channel, the hydrodynamic resistance of the fluid rises sharply reducing the mass flow. The hydrodynamic resistance in the microchannel is a few orders of magnitude smaller than the one in the nanochannels. That means it is nearly impossible to drive the flow and DNA samples into the nanochannels only by pressure, irrespective of the length of the DNA. Combining the capillary pressure and the electroosmotic particle flow seems to be a good solution.

The capillary and electroosmotic driven flow has been evaluated performing CFD (Computational Fluid Dynamics) simulations using the commercial software COMSOL. To account for the final thickness of the EDL (electrical double layer) on the electro-osmotic properties of the nanochannel, the electric forces acting on the double layer charge were modeled as a volume force on the liquid as ${ }^{10}$

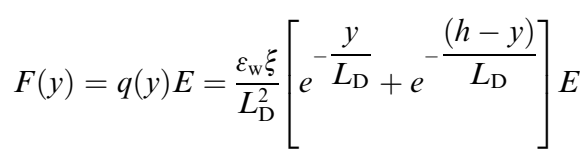

where $L_{\mathrm{d}}$ is the debye length, $\varepsilon_{\mathrm{w}}$ the permittivity of the medium, $\xi$ the zeta potential, $E$ the electric field, $h$ the channel height and $y$, respectively $(h-y)$, the distances to the bottom and top wall.

In this case the electrodes are L-shaped, with a short edge in the mainstream channel, and a longer edge leading into the nanochannel (Fig. 2a and b). The main stream in the microchannel is pressure driven. The particle flow to the nanochannel entrance and the flow profile in the channel were simulated. The flow profile in this case with exposed electrodes has a parabolic nature since the electrodes shorten the electric field (Fig. 2c), while the flow in the section without electrodes shows a EOF profile with zero condition on the wall rapidly growing to a maximum value over the electric double layer distance and then is either constant or slightly decreasing because of antiparabolic distortion due to backpressure (Fig. 2d).

To enhance the capillary filling, initially the channels were filled with ethanol, which has a very low contact angle (near to 0 degrees). After some ethanol washes, di-water solution with rhodamine (fluorescent marker) was introduced into the channels. 


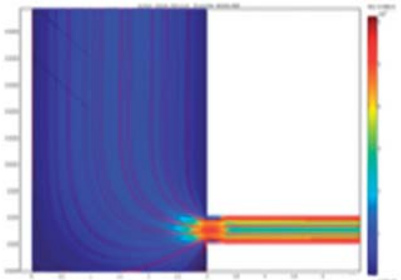

a

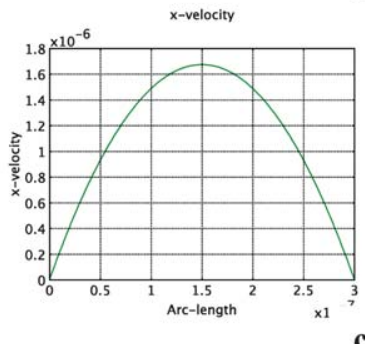

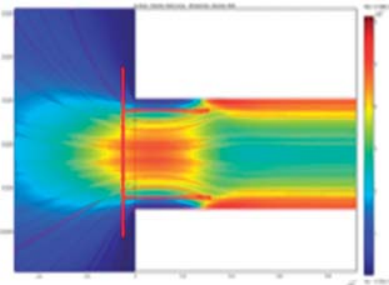

b

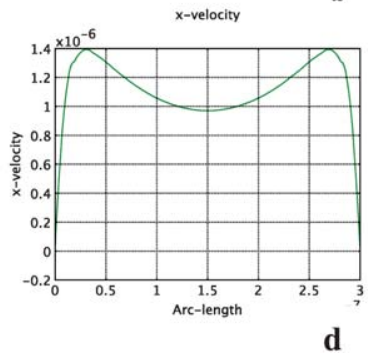

d
Fig. 2 (a) Simulation results showing micro- and nanochannels with flow directions, (b) diverting flow of the particles towards the nanochannel using electrodes, (c) simulation results showing the parabolic flow profile above the electrodes and (d) the EOF profile in a channel section without electrodes.

But while working with the DNA samples, ethanol cannot be used as it denatures the DNA. So, to suppress the electroosmotic flow and to have a free flow of the sample molecules, PVP is used. The nanochannels were flushed with PVP prior to the injection of the sample.

\section{Fabrication}

Extensive research is being carried out in constructing different nanochannels in order to manipulate single molecules to analyze, detect and separate them. Bio-medically this has vast applications. As of now molecules are identified and separated using electrophoresis and chromatographic techniques which are based on their mass to charge ratio. The sensitivity is far less with these techniques in single molecule detection unlike in nanochannels armed with electrodes.

There are different approaches to fabricate nanofluidic devices, by anodic bonding, ${ }^{9,11,12}$ using PDMS, ${ }^{13,14}$ etc. The electrodes involved in these devices are placed outside the nanochannels in the connecting microchannels to divert the biomolecules into the nanochannels, trap and analyze them. Our main aim in integrating electrodes within the nanochannels lies in studying the behaviour of the particles locally.

\section{Silicon-glass anodically bonded devices}

In this method, embedded titanium nitride electrodes and silicon carbide nanochannels were fabricated on a sodium contaminated glass wafer, whereas microchannels and access holes were fabricated on a DSP (double side polished) silicon wafer separately and then anodically bonded.

\section{Process flow for silicon-glass anodically bonded nanofluidic devices}

In the first step of the fabrication process, electrodes were fabricated followed by the patterning and etching of silicon carbide nanochannels. This process is described in detail in the following section (refer to Fig. 3).

\section{Fabrication of nearly flat 'TiN' electrodes}

This process was carried out on a sodium contaminated glass wafer. Firstly, the glass wafer was coated with aluminium $(600 \mathrm{~nm})$ on the backside to avoid the transparency of the glass wafer during processing.

Structures were patterned by standard photolithography and were dry etched $150 \mathrm{~nm}$ deep (Fig. 3c). Remaining photoresist was stripped off by standard cleaning methods and subsequently $200 \mathrm{~nm}$ of titanium nitride ( TiN) was sputtered at $350{ }^{\circ} \mathrm{C}$ on the etched patterns (Fig. 3d). Further, lithography was performed and the patterns with titanium nitride were covered with photoresist (Fig. 3f). By the etch back technique, titanium nitride from the inter-pattern spaces was completely etched away (Fig. 3g). Remaining photoresist was stripped off and the wafer was further cleaned with nitric acid followed by rinsing in diwater (Fig. 3h). By performing another lithography, the unpatterned part of the wafer was covered with photoresist (Fig. 3i) and excess TiN from the patterns was etched back until nearflatness was reached (Fig. 3k). The wafer was cleaned in nitric acid and rinsed with di-water (Fig. 31). The height of the patterns was measured using an alpha stepper and the wafer was cleaned with nitric acid and rinsed in di-water.

\section{Fabrication of silicon carbide nanochannels}

After the fabrication of nearly flat 'TiN' electrodes on the ' $\mathrm{Na}$ ' contaminated glass wafer, $100 \mathrm{~nm}$ silicon dioxide was deposited as an insulating layer followed by $150 \mathrm{~nm}$ of silicon carbide (Fig. 4a). Lithography was performed to open the bond pads (Fig. 4c). Silicon carbide followed by silicon dioxide was dry

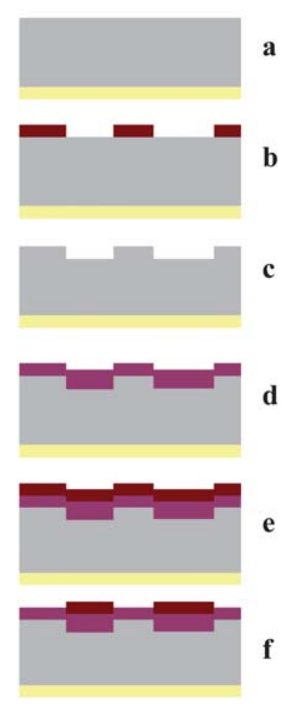

Glass wafer

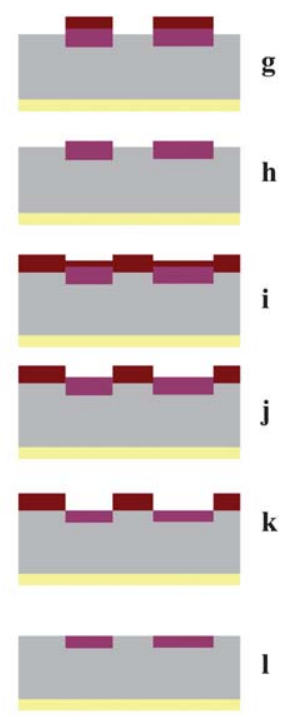

Photoresist

Titanium nitride 

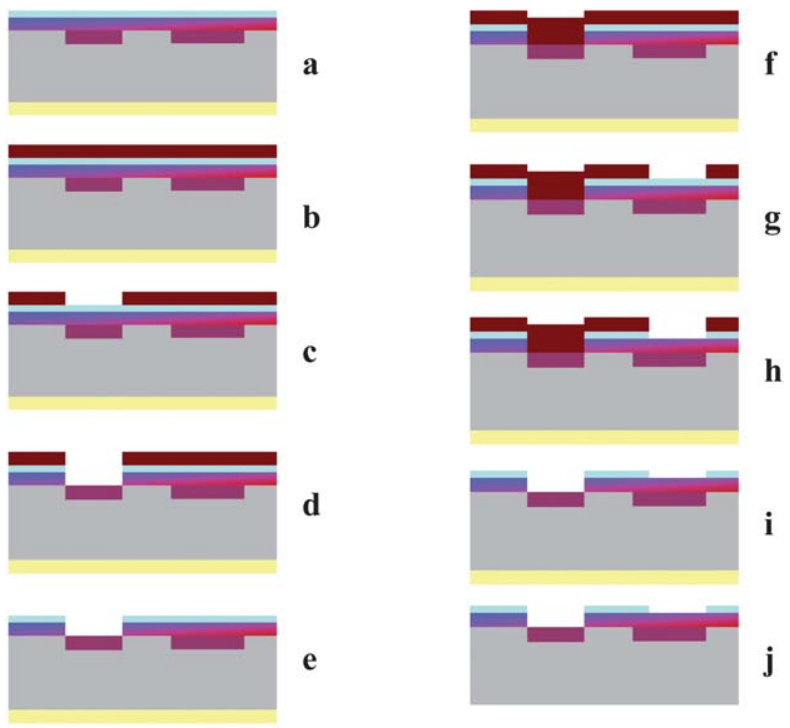

f
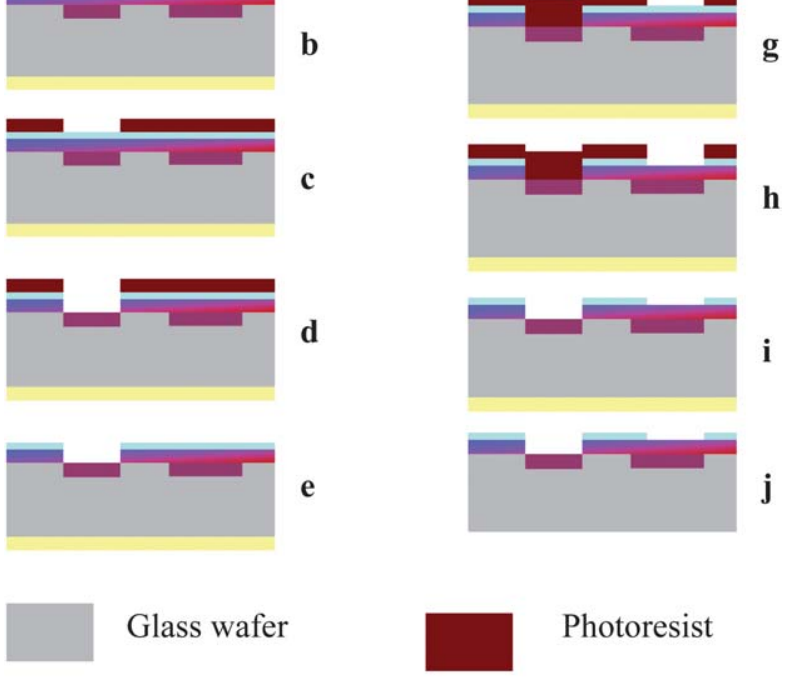

Photoresist

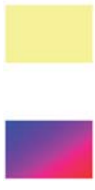

\section{Aluminium}

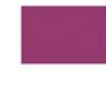

Titanium nitride

Silicon dioxide

Fig. 4 Flow chart for the fabrication of silicon carbide nanochannels.

etched until titanium nitride (Fig. 4d). The wafer was cleaned by standard cleaning methods (Fig. 4e) and lithography was performed to pattern the nanochannels (Fig. 4g).

Nanochannels were patterned on silicon carbide and were dry etched until silicon dioxide (Fig. 4h). Remaining photoresist was stripped and the wafer was cleaned by standard cleaning methods (Fig. 4i). Until this step, the glass wafer was covered with aluminium on the backside for reflection and sensing by the system during the etching processes. Once the channels were processed, aluminium from the backside of the wafer was stripped using aluminium etch (Fig. 4j).

\section{Fabrication of microchannels and access holes on a DSP silicon wafer}

On a DSP (double side polished) silicon wafer, micro-channels $(5 \mu \mathrm{m})$ and openings for bond pads and fluidic connections were patterned.

Initially microchannels were patterned by lithography and etched $(5 \mu \mathrm{m})$ in silicon (Fig. $5 \mathrm{~b})$. The wafer was cleaned by the standard cleaning method. A layer of $6 \mu \mathrm{m}$ silicon dioxide which is used as a masking material to etch through the silicon wafer completely was deposited. Lithography was performed to open the bond pads and access holes. Silicon dioxide was completely etched through the patterns (Fig. 5c). The backside of the wafer was covered with $4 \mu \mathrm{m}$ thick aluminium and $3 \mu \mathrm{m}$ thick photoresist as a protective membrane to withstand helium pressure during etching completely through the silicon (Fig. 5d). The wafer was further dry etched using an AMS Bosch etcher, to etch

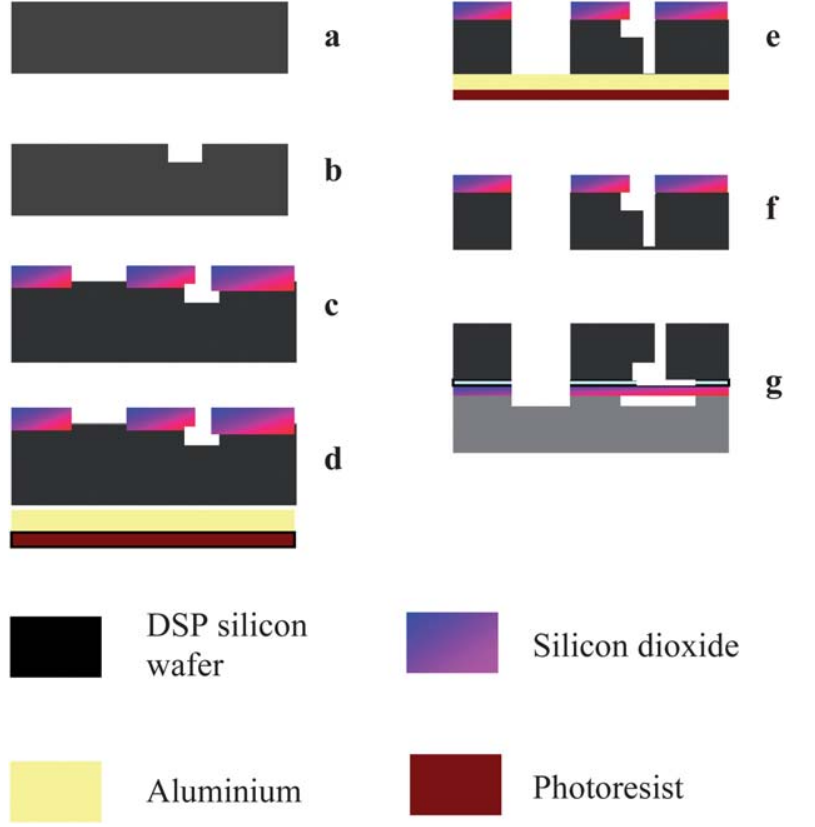

Fig. 5 Flow chart for the fabrication of DSP silicon wafer to open bond pads and access holes.

through completely (Fig. 5e). After etching ( 2 h), photoresist (by oxygen plasma), aluminium (by aluminium etch) and remaining silicon dioxide (using BHF) were stripped away (Fig. 5f). Both the wafers were bonded by anodic bonding (Fig. 5g).

\section{Anodic bonding between silicon and glass}

After the fabrication process, both the wafers were successfully bonded by anodic bonding.

Anodic bonding is a bonding process in which the substrates are bonded at elevated temperature $\left(400{ }^{\circ} \mathrm{C}\right)$ by placing and clamping the substrates between two metal electrodes. A high DC potential $(1000 \mathrm{~V})$ is applied between the electrodes creating an electrical field, which penetrates the substrates.

One substrate is glass that contains sodium ions, which at elevated temperatures are displaced from the bonding surface of the glass by the applied electrical field. The depletion of sodium ions near the surface of the glass makes the surface highly reactive with the silicon surface of the other substrate forming a solid chemical bond. The bonding is so strong that it is impossible to separate the wafers unless they are broken.

\section{Comparison between Chemical Mechanical Planarization (CMP) and the etch back technique}

CMP (Chemical Mechanical Planarization) is a process in the semiconductor industry used to planarize a wafer. This is a smoothing process of the wafer by a combination of chemical reaction and mechanical forces and so the name. Mechanical grinding alone causes too much damage and wet etching alone cannot attain good planarization. Originally CMP was used to planarize materials like silicon dioxide, but later on different chemicals and methods were introduced to polish even metals such as aluminium, tungsten, copper, etc. ${ }^{15,16}$ 
A typical CMP tool consists of a rotating platen that is covered by a pad. The wafer is mounted upside down in a carrier on a backing film. The retaining ring keeps the wafer in the correct horizontal position. Both the platen and the carrier are rotating. Good speed control is important. The carrier is also oscillating. For loading and unloading, a robot system is installed. During loading and unloading, the wafer is kept in the carrier by vacuum. During chemical mechanical polishing, pressure is applied by down force on the carrier, transferred to the carrier through the carrier axis and a gimbal mechanism. Besides that gas pressure or back pressure is also loaded on the wafer. High points on the wafer are subjected to higher pressures compared to lower points, hence, the removal rates are enhanced and planarization is achieved. The slurry is supplied from above on the platen. The grain size, the material of the abrasive component and the $\mathrm{pH}$ control of the slurry are process relevant. Normally alkali conditions are used.

Fabrication of nanochannels by CMP was very well defined in the literature. By this method the length, width and height of the channels can be controlled without applying any lithography techniques. ${ }^{16}$ The dimensions of the channels defined in this literature are in the range of $25 \mathrm{~nm}$ wide, $100 \mathrm{~nm}$ deep and $10 \mathrm{~mm}$ long.

We have initially tried CMP as well as pure mechanical polishing to planarize the surface having titanium electrodes, fabricated on silicon and glass wafers. In our case, even though the length of the channel corresponds somewhat to the above mentioned (10 $\mathrm{mm}$ long) dimensions the depth varied largely with the width of the channels. It was difficult to retain the original patterns with metal after the CMP process. There was highly visual damage on the metal electrodes and interconnects. The planarization process was uneven for different structures in our design, which range from $300 \mu \mathrm{m}$ to $10 \mu \mathrm{m}$ in width.

A possible explanation for this problem could be most likely from the varying dimensions in our designs. After several trials it was decided to proceed further through a different mechanism, the etch-back technique.

\section{Etch-back technique}

Etch-back is a technique in which the metal on the electrodes (in this case titanium nitride) after sputtering will be thinned down to flat/near-flat thickness. This process is slightly laborious compared to the CMP process but is reliable when using metals which have a lower etch rate. We have opted for titanium nitride, which in recent times is frequently used in the semiconductor industry. It has a dry etch rate of approximately $5 \mathrm{~nm} \mathrm{~s}^{-1}$, which is in a near controllable range. Fig. 3 explains the etch back process in detail.

\section{Working of the device}

The device has 2 microchannels with inlet and outlets. Nanochannels are bridged between the 2 microchannels. Nanochannels are in turn embedded with electrodes. The latest devices also have electrodes in the micro/nanochannel junctions to facilitate the flow of the particles/DNA into the nanochannels when the voltage is applied. The device will be mounted onto a holder pre-defined with electrical and fluidic connections.
Initially the channels will be filled with rhodamine + di-water, so that they can be observed clearly under the microscope. The DNA solution is pumped through the inlet of the microchannel. Once the channels are filled, coiled DNA tagged with a dye (BOBO) can be observed circulating within the microchannels. Once a voltage is applied the coiled DNA is expected to concentrate near the entrance of the nanochannels. Further increase in voltage should lead the coiled DNA into the nanochannel, uncoiling itself slowly.

\section{Device holder}

The front view of the holder is shown in Fig. 6. The chip is clamped in the center recess by the fixing plate with the access holes facing down to the liquid interface on the bottom plate. The o-rings are placed in between, to achieve tight sealing.

The fluid connection is realized by connecting the plastic tubes to the backside of the holder. The electrical connection is realized by a double clamping system. The chip is connected to the PCBs via rubber connectors in which the conductive and nonconductive strips are alternatively stacked. The double clamping system makes it possible to connect both the top and the bottom layer of the microfluidic chip along with the freedom to connect either the top or the bottom layer of the chip.

\section{Measurement setup}

The fabricated device has to be tested thoroughly prior to the experiments with DNA. Rhodamine + ethanol + di-water was used to test for any leakage from the fabricated micro- and nanochannels after anodic bonding. The working of the integrated electrodes was checked using carboxylate modified fluorescent polystyrene beads. This process is explained in detail in the next section.

The fluorescent polystyrene (PS) beads used in these experiments are carboxylated-modified fluospheres from Molecular Probes (USA). The bead's excitation and emission wavelengths are $580 \mathrm{~nm}$ and $630 \mathrm{~nm}$, respectively. The holder containing the microfluidic chip was mounted onto the table of an inverted microscope (IX71, Olympus) equipped with a low noise selfcooling CCD camera (ColorView II, Olympus). The images or

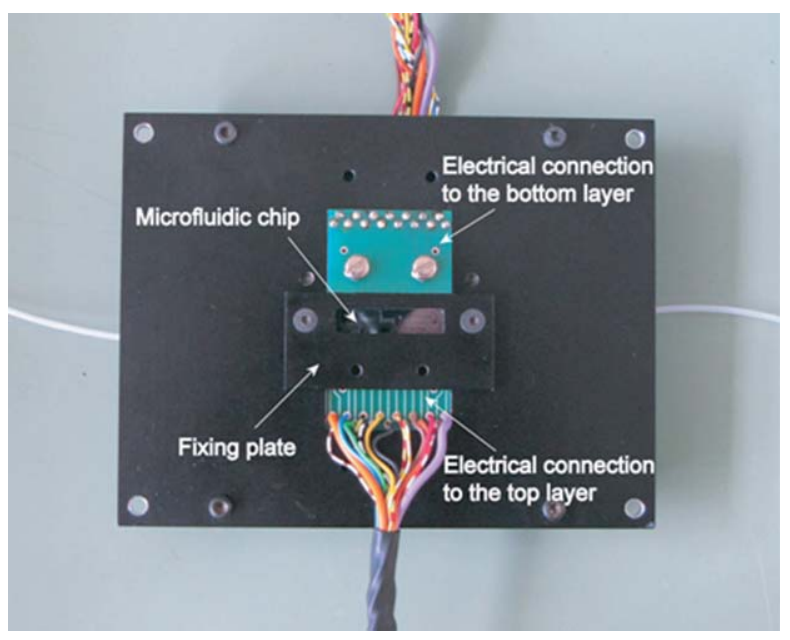

Fig. 6 Front view of the device holder. 
the videos were acquired by an imaging software (analySIS docu, Olympus) for further data processing and analysis. To see the fluorescent image, an excitation filter $(\lambda=570-590 \mathrm{~nm})$ is used in combination with an emission filter $(\lambda=600-660 \mathrm{~nm})$, both from Olympus. To apply the electric fields, AC supplies (HP33120A arbitrary waveform generator) were connected to the chip via the electrical interface on the holder. In order to check the applied voltage, an oscilloscope (HP54601B) was used. A continuous flow was generated by the pressure difference between the inlet and the outlet. The flow velocity was controlled by tuning the height difference of the liquid surface levels in the reservoirs.

After several experiments, many PS particles that are adhered to the surface of the wall will be left in the channel. Toluene was found capable of dissolving the PS particles. Therefore, flushing the channel with toluene 2-3 times will effectively remove all the residual PS particles. After cleaning with di-water, the device could be re-used many times and no deterioration in the performance was observed.

\section{Testing of nanochannels using rhodamine and polystyrene beads}

Devices were fabricated with varying nanochannel depths viz. 100, 230 and $400 \mathrm{~nm}$. The device was clamped to the holder and in turn placed on an Olympus inverted microscope. Initially, ethanol was supplied to the nanochannels through the inlet to reduce the surface tension. Once the channels were filled completely with ethanol, di water was supplied and the channels were successfully filled. Rapid evaporation of fluids can be observed within the nanochannels and therefore continuous supply is needed. Continuous flow is supplied with the help of fluidic connections which are connected to the holder. Care was always taken to checkfor any leakage while supplying the liquid.

The channels were successfully checked for any leakage by filling with rhodamine + ethanol + di-water (Fig. 7a and b). After successfully testing the nanochannels, carboxylate modified fluorescent polystyrene beads of the order $200 \mathrm{~nm}$ were used to test the working of the electrodes.

Once the nanochannels were filled with ethanol, beads were injected into the inlet of the microchannel with the help of a syringe. Electrodes were switched "on" to facilitate the movement of polystyrene beads into the nanochannels. Optical inverted microscope images show the trapped polystyrene beads within $50 \mu \mathrm{m}$ and $15 \mu \mathrm{m}$ wide channels (Fig. 7c and d). The voltages applied are in the range of 3-12 volts.

\section{Experiments with DNA}

After testing the device for the proper working of the nanochannels and integrated electrodes, further experiments were conducted using DNA molecules. The idea to study a single DNA molecule within the nanochannels has been practiced for many years. Several researchers reported their findings on DNA mobility, ${ }^{17}$ DNA molecule trajectories by di-electrophoresis, ${ }^{18}$ and field-dependent DNA mobility in nanoslits, ${ }^{19}$ are a few to mention.

Our simple experiment on DNA tracking within a nanochannel provides an insight regarding the time taken by a DNA molecule to travel through a nanochannel in relation to the
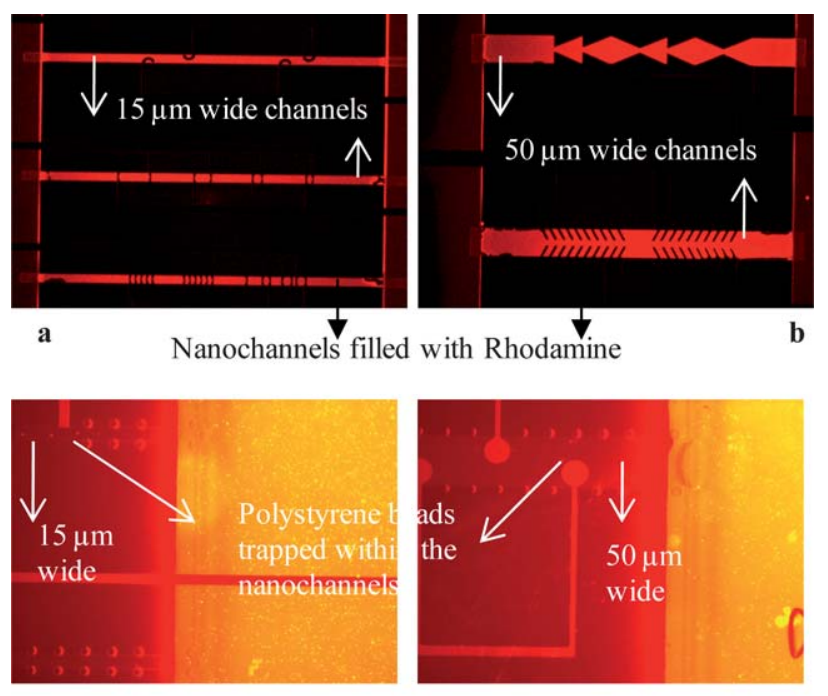

c

d

Fig. 7 (a and b) Microscopic images (fluorescent inverted mode) showing $15 \mu \mathrm{m}$ and $50 \mu \mathrm{m}$ wide nanochannels filled with rhodamine/ ethanol/di-water solution (bright red color). (c and d) $15 \mu \mathrm{m}$ and $50 \mu \mathrm{m}$ wide nanochannels with trapped fluorescent polystyrene beads.

length, depth, width of the channel and the voltage applied. The materials required for the experiments are results from different literature studies. ${ }^{19,4}$ In the following section, the materials used and the experimental protocol are discussed in detail.

\section{Materials required}

(a) DNA

(b) Dye (BOBO and JOJO)

(c) TAE buffer (Tris base + acetic acid + ethylenediaminetetraacetic acid (EDTA))

(d) $\beta$-Mercaptoethanol

(e) PVP (polyvinylpyrrolidone)

a: In our experiments two types of DNA have been used, $\lambda$ DNA (unmethylated) and T4GT7 DNA which were acquired from Promega, USA.

$\lambda$ DNA: comes from a virus called phage Lambda, which is harmless to humans and makes an excellent and safe source of DNA. $\lambda$ DNA is around $48 \mathrm{kbp}$ long.

T4GT7 DNA: is a derivative of bacteriophage T4 and is around $166 \mathrm{kbp}$ long.

Both the DNAs have to be stored at $-200^{\circ} \mathrm{C}$ when not in use. b: Dye: BOBO and JOJO (Invitrogen, USA) dyes were used to tag DNA molecules and conduct the fluorescent measurements.

BOBO-3 iodide: emission and excitation wavelengths are $570 / 602$.

JOJO-1 iodide: emission and excitation wavelengths are $529 / 545$.

c: TAE buffer: TAE buffer solution contains a mixture of Tris base + acetic acid+ EDTA. Conventionally it is used in agarose electrophoresis for the separation of nucleic acids. TAE has a lower buffer capacity than TBE (Tris base + boric acid + EDTA). DNA runs faster in TAE than TBE.

$\mathrm{d}$ : $\beta$-Mercaptoethanol: is also called 2-mercaptoethanol. It is a colorless liquid and considered as toxin causing irritation in the respiratory tract upon inhalation because of its strong odour. 
Severe exposure may lead to death. In our experiment $\beta$-mercaptoethanol $(3 \%)$ is used to suppress photobleaching.

Photobleaching is a phenomenon in which the observation of fluorescent molecules through a microscope will be eventually destroyed by continuous light exposure.

e: PVP (poly vinyl pyrrolidone): PVP is a light flaky powder which is soluble in water and other polar solvents. As a solution, PVP has excellent wetting properties and readily forms films. PVP suppresses the electroosmotic flow and therefore it is used in combination with DNA.

(In general ethanol can be used to reduce the surface tension within nanochannels, but with biological molecules ethanol is not feasible as it denatures the molecules.)

\section{Preparation of DNA sample solution}

TAE buffer $(10 \times$ standard $)$ is diluted to $1 \times$ using di-water. DNA and dye $(\mathrm{BOBO})$ in the ratio of $8: 1$ were added to $1 \times$ TAE buffer and the solution was incubated for $1 \mathrm{~h}$. 3\% $\beta$-Mercaptoethanol and 2.5\% PVP were added to the diluted DNA solution and again incubated for $1 \mathrm{~h}$ at room temperature. When not in use the solution was stored at $-20{ }^{\circ} \mathrm{C}$.

\section{Experiment}

Initially with the help of a syringe, a solution of rhodamine + diwater was injected into the device (channels filled with rhodamine can be clearly observed under fluorescent mode). Once filled with rhodamine + di-water solution, the device was left for a few hours so that the channels will be completely filled with the solution and can clearly be observed under the fluorescent microscope (Fig. 8).

Once the channels were tested for clear observation, the device was clamped onto the holder and in turn the whole setup was mounted on an inverted microscope. The channels were initially filled with PVP $+\beta$-mercaptoethanol solution (reduction in fluorescence was observed within the channels resulting in a probable reaction of $\beta$-mercaptoethanol with rhodamine). With the help of a syringe, the DNA solution was pumped into the inlet of the device through the fluidic connections attached to the holder. After a certain time, coiled, free circulating DNA can be observed within the microchannels.

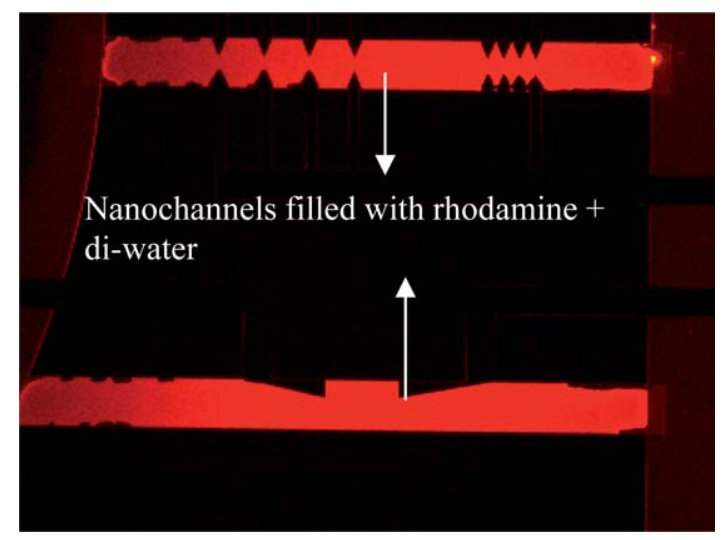

Fig. 8 Nanochannels filled with rhodamine + di-water before DNA solution was introduced.

\section{DNA tracking in a nanochannel}

The devices were fabricated to study the DNA molecule in a nanochannel and further experiments were carried out to track a DNA molecule within a nanochannel with embedded electrodes.

For these experiments two solutions were prepared:

Solution 1. 3\% $\beta$-Mercaptoethanol $+2.5 \%$ PVP.

Solution 2. Unmethylated $\lambda$ DNA (Promega, USA) was treated with the JOJO dye in the ratio of $8: 1$ (DNA : dye) in the presence of $1 \times$ TAE buffer. 3\% $\beta$-Mercaptoethanol and $2.5 \%$ PVP were added to the DNA solution and incubated for $1 \mathrm{~h}$ at room temperature.

Firstly the device was clamped onto the holder corresponding to the electrical and fluidic connections and in turn the whole setup was placed on an Olympus inverted microscope. With the help of a syringe, solution 1 was initially injected into the microchannel of the device through the inlet. PVP reduces the surface tension of the nanochannels and allows the free flow of solution whereas $\beta$-mercaptoethanol reduces photobleaching. Once the channels were completely filled with solution 1, solution 2 was injected through the inlet of the microchannel. The flow of solution with coiled DNA samples can be seen within the microchannels.

Microchannels are equipped with electrodes close to the entrance of the nanochannel. These electrodes work as pre-sorter electrodes in diverting the far-off molecules towards the entrance of the nanochannel.

Once the flow of DNA molecules was inside the microchannel, a potential of 4 volts was supplied to the electrodes in the microchannel (Fig. 9). The negatively charged molecules were attracted to the positive electrode by electrophoresis, which helps in bringing the DNA molecules close to the entrance of the nanochannel. Then a second voltage source $(12 \mathrm{~V})$ connected between the microchannels was switched "on". This causes an electric field in the nanochannels which facilitates the DNA molecule to enter in two ways, via the generation of an electroosmotic flow towards the entrance and an electrophoretic force acting on the negatively charged DNA molecule.

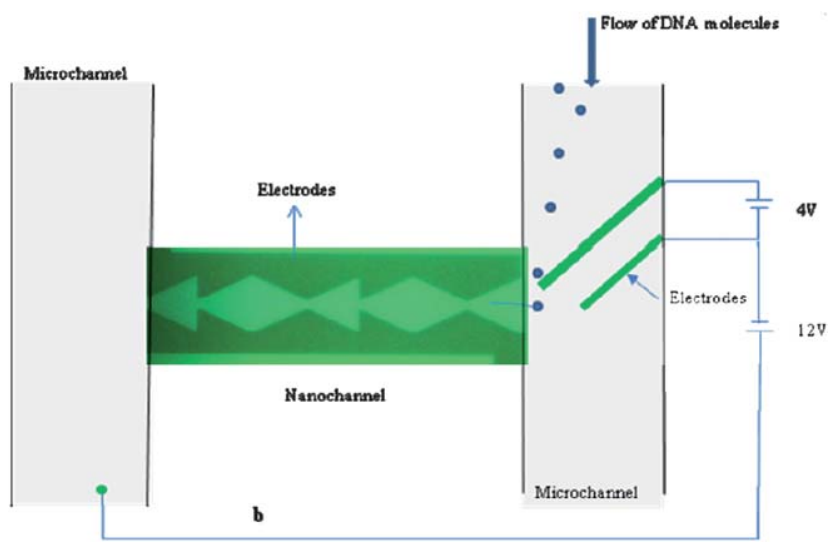

Fig. 9 Schematic representation of the working of electrodes in the micro- and nanochannel of the device. 
The electrodes are insulated from the fluid by a layer of silicon dioxide and therefore the influence on the electric fields in the channel will be minimal, although there might be an effect on the double layer charge and so on the uniformity of the electroosmotic flow throughout the nanochannel. As the channel walls are negatively charged ${ }^{6}$ as well as the DNA, electrostatic repulsion from the channel walls helps the particles to concentrate at the centre of the channel. The maximum voltage applied at this stage to drag the molecule into the nanochannel was $12 \mathrm{~V}$. The voltage range is limited with respect to the breakdown voltage of the insulating layer.

The actual length of the nanochannel is $600 \mu \mathrm{m}$, of which $25 \mu \mathrm{m}$ at each end of the channel was overlapping with the microchannel (to avoid any alignment related issues during fabrication). DNA travelled with a velocity of $6.3 \mu \mathrm{m} \mathrm{s}^{-1}$ (average of 5 frames) to cross the channel of $600 \mu \mathrm{m}$ length in $94 \mathrm{~s}$. Only 5 frames were possible for the respective molecule in order to trace, track, picture and process the images using Analysis software.

Analyzing the data, it was observed that the velocity of the DNA molecule was very low at the ends of the channel in comparison with the velocity at the centre. Initially, it took a lot of time for the DNA to enter into the nanochannel (time not calculated) (Fig. 10a). Once the DNA is in the channel the velocity is accelerated in such a way that the molecule travelled approx. $200 \mu \mathrm{m}$ in $25 \mathrm{~s}$. It is assumed that the intensity of the electric field was quite weak at the entrance of the nanochannel, which delays the entry of the DNA molecule into the channel. Once the DNA is within the nanochannel, due to higher intensity of the electric field, a rapid rise in the velocity of the molecule was observed. In just $12 \mathrm{~s}$ (the time taken to capture and process the
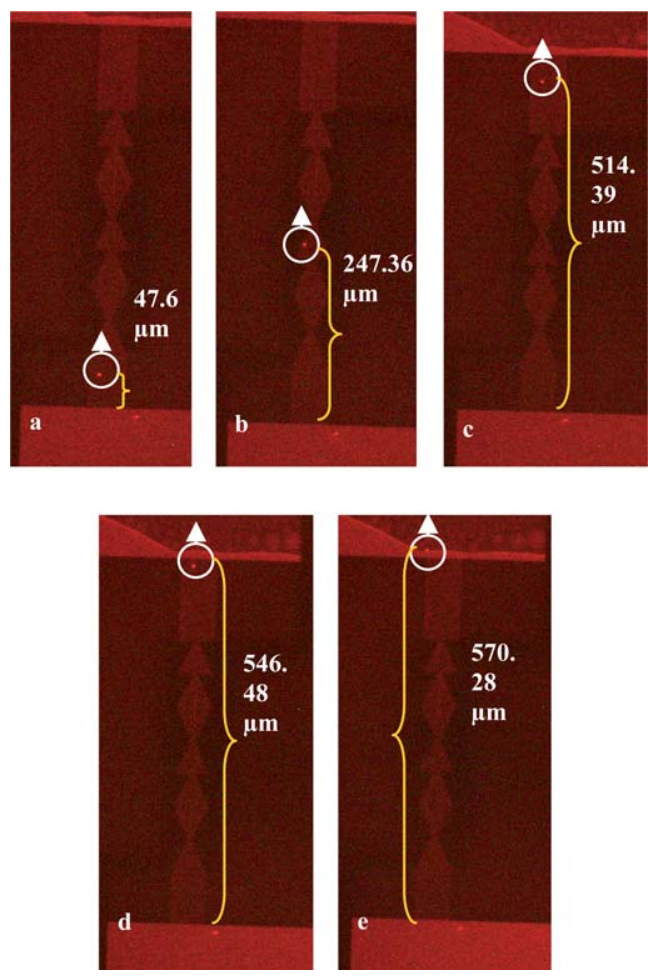

Fig. 10 Photographs showing the DNA path with the distance calculated from the entrance of the channel.
Table 1 The distance travelled by DNA from point to point in a certain time period

\begin{tabular}{llll}
\hline Fig. 7 & Duration/s & $\begin{array}{l}\text { Distance from } \\
\text { point to point/ } \mu \mathrm{m}\end{array}$ & Total distance \\
\hline a & & 47.06 & 47.6 \\
a and b & 25 & 199.76 & 247.6 \\
b and c & 12 & 267.03 & 514.39 \\
c and d & 33 & 32.09 & 546.48 \\
d and e & 24 & 23.08 & 570.28 \\
\hline
\end{tabular}

frame) the molecule travelled $267.03 \mu \mathrm{m}$ (Fig. 10c), through the center part of the nanochannel.

Fig. 10d and e show the loss in velocity, which clearly depicts that the intensity of the electric field that was acting on the DNA molecule was quite low. Table 1 shows the distance travelled by DNA from point to point and total distance in a certain time, in five different frames.

\section{Explanation and conclusion}

DNA after reaching the junction of the microchannel and nanochannel needs some time to adapt to the shape of the channel and enter into the nanochannel. The electric field acting on DNA at this stage is relatively weak and as a result it takes some time for the molecule to get dragged into the nanochannel.

Once the DNA is completely within the nanochannel, where the maximum electric field exists, it is accelerated to a higher velocity which is directly proportional to the value of the electric field that is being applied on the DNA molecule. This higher speed is due to two effects, the increase in electroosmotic flow speed and the electrophoretic force on the particle.

Reaching the other end of the channel, where the nanochannel overlaps with the microchannel, the electric field strength drops quickly and so does the particle speed. To summarize, the field intensity and the mobility within the nanochannel were higher at the centre in comparison to those at the beginning and at the ends. This corresponds with the observed higher DNA speed in the center part of the nanochannel.

\section{Acknowledgements}

The authors would like to thank the Foundation for Fundamental Research on Matter (FOM) for supporting and funding this work. Special thanks will be extended to Dr Heidi Dietrich and Dr Gea Parikesit for providing the DNA and dye samples. We also would like to thank Dr Jaber Derakhshandeh from ECTM, Delft University of Technology, for helping with the CMP experiments. We also would like to thank our former colleague Dr Lujun Zhang for the pictures of the device holder. Finally, the authors would like to thank the staff of the DIMES clean room for their technical support.

\section{References}

1 W. Sparreboom, A. van den Berg and J. C. Eijkel, Transport in nanofluidic systems: a review of theory and applications, New J. Phys., 2010, 12, 015004, 23p.

2 P. Abgrall and N. Nguyen, Nanofluidic devices and their applications, Anal. Chem., 2008, 80, 2326-2341.

3 R. Qiao and N. R. Aluru, J. Chem. Phys., 2003, 118, 4692. 
4 W. D. Volkmuth and R. H. Austin, Nature, 1992, 358, 600-602.

5 J. Han and H. G. Craighead, J. Vac. Sci. Technol., A, 1999, 17, $2142-$ 2147.

6 R. Karnik, R. Fan, M. Yue, D. Y. Li, P. D. Yang and A. Majumdar, Nano Lett., 2005, 5, 943-948.

7 M. B. Stern, M. W. Geis and J. E. Curtin, J. Vac. Sci. Technol., B: Microelectron. Nanometer Struct.-Process., Meas., Phenom., 1997, 15, 2887-2891.

8 H. gardeniers and A. van den Berg, Micro- and nanofluidic devices for environmental and biomedical applications, Int. J. Environ. Anal. Chem., 2004, 84(11), 809-819.

9 V. G. Kutchoukov, L. Pakula, G. O. F. Parikesit, Y. Garini, L. K. Nanver and A. Bossche, Fabrication of nanofluidic devices in glass with polysilicon electrodes, Sens. Actuators, A, 2005, 123-124, 602-607.

10 H. Bruus, Theoretical Microfluidics, 2007, Oxford University press.

11 A. Datta, et al., Nanofluidic channels by anodic bonding of amorphous silicon to glass to study ion-accumulation and iondepletion effect, Talanta, 2006, 68, 659-665.

12 N. Pussadee, Poly (dimethylsiloxane) Based Micro- and Nanofluidic Device Fabrication for Electrophoresis Applications, The Ohio state University, 2010, vol. 133, p. 3435645.

13 S.-m. Park, et al., A method for nanofluidic device prototyping using elastomeric collapse, Proc. Natl. Acad. Sci. U. S. A., September 15, 2009, 106(37), 15549-15554.
14 Y. Awano, Carbon Nanotube (CNT) Via Interconnect Technologies: Low temperature CVD growth and chemical mechanical planarization for vertically aligned CNTs, Proc. 2006 ICPT, 2006, p. 10.

15 S. Wolf, Silicon Processing for the VLSI Era, Deep-submicron Process Technology, 2002, ch. 8, Chemical mechanical polishing, vol. IV, pp. 313-432.

16 Choonsup Lee, E. H. Yang, N. V. Myung and T. George, A nanochannel fabrication technique using chemical-mechanical polishing (CMP) and thermal oxidation, IEEE Nano, 2003, 2, $553-$ 556.

17 J. O. Tegenfeldt, et al., Proc. Natl. Acad. Sci. U. S. A., 2005, 101, 10979.

18 G. O. F. Parikesit, A. P. Markesteijn, O. M. Piciu, A. Bossche, J. Westerweel, I. T. Young and Y. Garini, Size-dependent trajectories of DNA macromolecules due to insulative dielectrophoresis in submicrometer-deep fluidic channels, Biomicrofluidics, 2008, 2, 024103.

19 G. B. Salieb-Beugelaar, J. Teapal, J. van Nieuwkasteele, D. Wijnperle, J. O. Tegenfeldt, F. Lisdat, A. van den Berg and J. C. T Eijkel. Fielddependent DNA mobility in $20 \mathrm{~nm}$ high nanoslits. Nano Lett., 2008, 8, (no. 7). pp 1785-1790.

20 V. Tabard-Cossa, D. Trivedi, M. Wiggin, N. N. Jetha and A. Marziali, Noise analysis and reduction in solid-state nanopores, Nanotechnology, 2007, 18, 305505. 